\title{
Infrared Reflectance Kramers-Kronig Analysis by Anchor-Window Technique
}

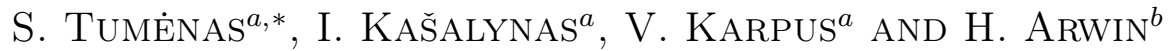 \\ ${ }^{a}$ Center for Physical Sciences and Technology, Semiconductor Physics Institute \\ A. Goštauto 11, 01108 Vilnius, Lithuania \\ ${ }^{b}$ Department of Physics, Chemistry and Biology, Linköping University \\ 58183 Linköping, Sweden
}

\begin{abstract}
An algorithm for the Kramers-Kronig analysis of the reflectivity spectra, based on an anchor-window technique is presented. The high-frequency asymptote, required for the Kramers-Kronig analysis, is determined by minimizing differences between the Kramers-Kronig-deduced optical constants of a system under investigation and known optical constants measured in a small anchor-window. The algorithm is illustrated by applying it for a reconstruction of the optical conductivity $\sigma(\omega)$ of the fci-ZnMgRE quasicrystals in the spectral range of $0.01-6.5 \mathrm{eV}$ from the experimental IR Fourier-transform reflectivity data and the experimental spectral ellipsometry VIS-UV data. The reliability of the suggested Kramers-Kronig analysis technique is confirmed by independent infrared spectral ellipsometry $\sigma(\omega)$ measurements for fci-ZnMgRE.
\end{abstract}

PACS: 71.23.Ft, 78.20.Ci

\section{Introduction}

The infrared reflectance spectroscopy is a main tool for optical investigations in the IR spectral range. The optical parameters of a system under investigation, the dielectric function $\varepsilon(\omega)$ or the optical conductivity $\sigma(\omega)$ (which is directly related with the dielectric function as $\varepsilon(\omega)=1+\mathrm{i} 4 \pi \sigma(\omega) / \omega)$ are determined from the reflectivity spectrum $R(\omega)$ making use of the Kramers-Kronig (KK) relations. The KK analysis, however, requires for extrapolations of the experimental data to the low- and high-frequency limits, which usually essentially reduces an accuracy of the analysis.

We present an algorithm of the Kramers-Kronig analysis based on an anchor-window technique. The high-frequency asymptote of the reflectivity spectrum is determined by a minimization of differences between the KK-deduced optical conductivity $\sigma_{\mathrm{KK}}(\omega)$ and the known $\sigma(\omega)$ values, measured a priori in a small anchor-window. The algorithm is illustrated by reconstructing the wide-range, 0.01-6.5 eV, optical conductivity spectrum of fci$-\mathrm{ZnMgRE}(\mathrm{RE}=\mathrm{Y}, \mathrm{Ho})$ quasicrystals.

\section{Experimental}

The single-grain face-centred icosahedral (fci) $\mathrm{Zn}_{62} \mathrm{Mg}_{29} \mathrm{Y}_{9}$ and $\mathrm{Zn}_{65} \mathrm{Mg}_{25} \mathrm{Ho}_{10}$ quasicrystals were grown by the liquid-encapsulated top-seeded solution-growth method [1]. Prior to each optical measurement, the optical surfaces of samples were mechanically

\footnotetext{
* corresponding author; e-mail: tumenas@pfi.lt
}

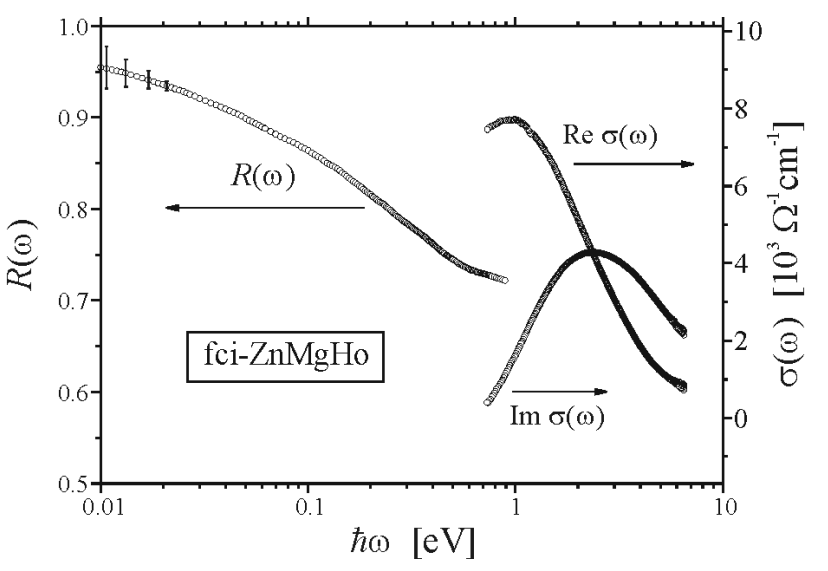

Fig. 1. Experimental fci-ZnMgHo reflectivity $R(\omega)$ and the optical conductivity $\sigma(\omega)$ spectra.

grind and fine-polished with a diamond paste $(10 \mu \mathrm{m}$, $5 \mu \mathrm{m}, 1 \mu \mathrm{m}$, and $0.25 \mu \mathrm{m}$ ) and an alumina suspension (0.02 $\mu \mathrm{m})$.

The IR reflectivity $R(\omega)$ spectra (Fig. 1) in the spectral range $0.01-0.9 \mathrm{eV}$ were measured by Fourier transform spectrometer Nicolet 8700. The VIS-UV optical conductivity $\sigma(\omega)$ spectra (Fig. 1) in the spectral range $0.73-6.5 \mathrm{eV}$ were measured by spectroscopic ellipsometry (SE) technique by the rotating analyzer ellipsometer VASE (J.A. Woolam Co, Inc.). Appending the IR reflectivity data by the $R(\omega)$, calculated from the SE VIS-UV data, we obtained the reflectivity spectra in the spectral range $0.01-6.5 \mathrm{eV}$ (see Fig. 2). 


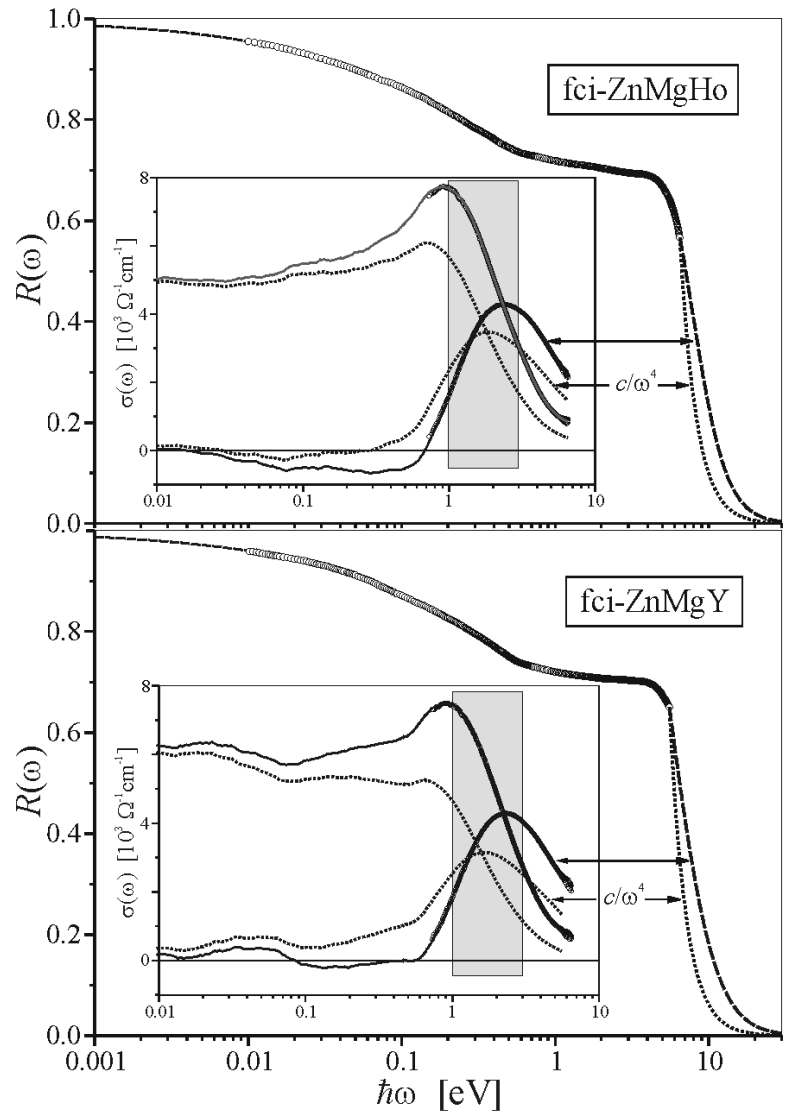

Fig. 2. The experimental reflectivity $R(\omega)$ spectra (dots) and extrapolations by the standard high-frequency asymptote $R_{\mathrm{hf}}(\omega)=c / \omega^{4}$ (dotted curves) and by the anchor-window determined asymptote (dashed curves). Insets present the experimental optical conductivity $\sigma(\omega)$ spectra (dots) and the KK-deduced $\sigma_{\mathrm{KK}}(\omega)$ spectra (dotted and dashed curves). Shaded areas in insets indicate the anchor-window.

\section{Algorithm of the anchor-window technique}

In metallic compounds, the low- and high-frequency reflectivity asymptotes, required for the Kramers-Kronig analysis, are usually approximated by the relations

$$
R_{\mathrm{lf}}(\omega)=1-c \sqrt{\omega}, \quad R_{\mathrm{hf}}(\omega)=\frac{c}{\omega^{4}} .
$$

Here the low-frequency asymptote $R_{\mathrm{lf}}(\omega)$ corresponds to the Hagen-Rubens law and the high-frequency asymptote $R_{\mathrm{hf}}(\omega)$ corresponds to a generic frequency dependence, which settles down at photon energies exceeding characteristic energies of a system under investigation. Making use the $R(\omega)$ extrapolations (1) and of the Kramers-Kronig relation

$$
\theta(\omega)=-\frac{\omega}{\pi} P \int_{0}^{\infty} \mathrm{d} \omega^{\prime} \frac{\ln R\left(\omega^{\prime}\right)}{\omega^{\prime 2}-\omega^{2}},
$$

one can calculate the phase factor $\theta(\omega)$ of the complex reflection amplitude $r(\omega)=|r(\omega)| \exp (\mathrm{i} \theta(\omega))$. Then, making use of the relation $r(\omega)=\left[\varepsilon^{1 / 2}(\omega)-1\right] /\left[\varepsilon^{1 / 2}(\omega)+1\right]$, one can determine the dielectric function and the optical

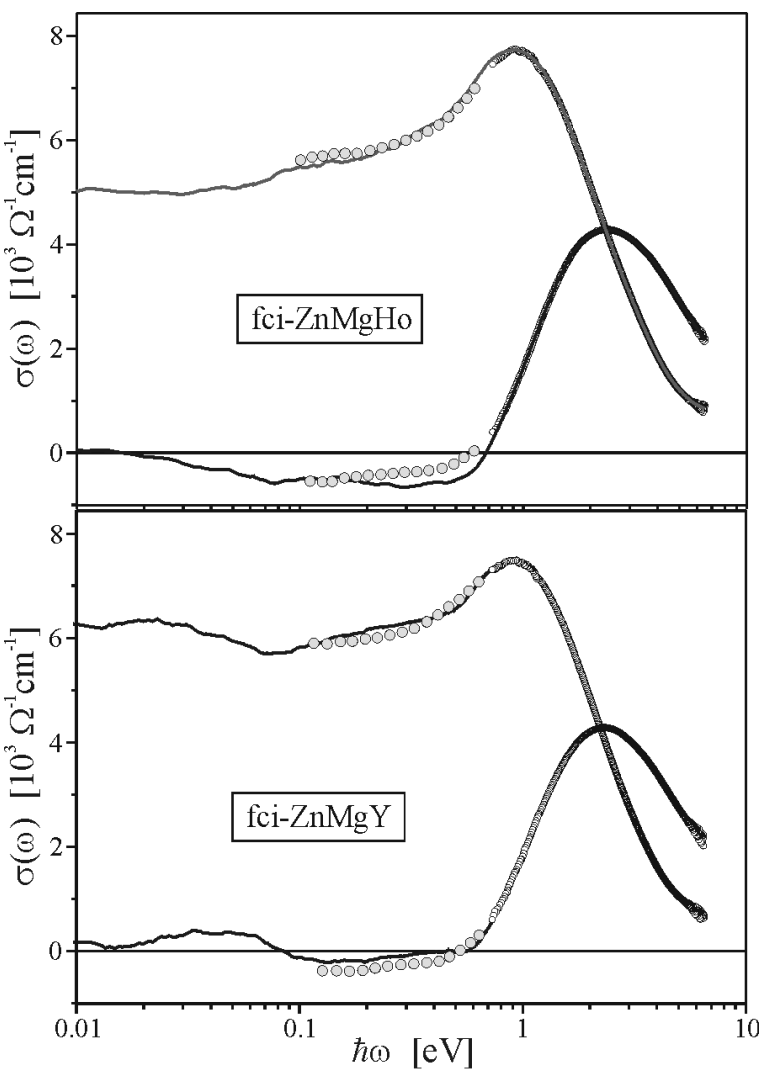

Fig. 3. Experimental optical conductivity $\sigma(\omega)$ spectra (dots) and the $\sigma(\omega)$ spectra deduced by the Kramers-Kronig analysis (curves).

conductivity. Results of the calculations are presented in insets to Fig. 2 by dotted curves. As seen, the optical conductivity $\sigma_{\mathrm{KK}}(\omega)$, deduced by the Kramers-Kronig analysis, essentially differs from experimental $\sigma(\omega)$ spectra, presented by dots in Fig. 2 insets.

There are various techniques to improve an accuracy of the KK analysis [2]. We suggest an anchor-window technique, which is a modification of the anchor-point method [3]. The high-frequency asymptote is modelled by the inverse polynomial

$$
R_{\mathrm{hf}}(\omega)=\left[b_{0}+b_{1} \omega+b_{2} \omega^{2}+b_{3} \omega^{3}+b_{4} \omega^{4}\right]^{-1},
$$

the coefficients $b_{i}$ of which are determined by minimizing differences between the KK-deduced optical conductivity and the experimental $\sigma(\omega)$ values, known in a narrow spectral region - the anchor-window. The error function was chosen in the form

$$
\chi=\frac{1}{N} \sum_{n=1}^{N}\left|\frac{\sigma_{\mathrm{KK}}[n]-\sigma_{\text {exper }}[n]}{\sigma_{\text {exper }}[n]}\right|^{2},
$$

where $\sigma_{\text {exper }}[n]$ is the experimental optical conductivity value at the spectral point $n$, which belongs to the anchor-window, $\sigma_{\mathrm{KK}}[n]$ is the KK-deduced value, and $N$ is the number of points within the anchor-window. The low-frequency asymptote of the reflectivity spectrum was approximated by the relation 


$$
R_{\mathrm{lf}}(\omega)=1-a_{1} \sqrt{\omega}-a_{2} \omega .
$$

The $a_{1}$ and $a_{2}$ coefficients were determined by the least-squares technique, minimizing the error function

$$
\chi=\frac{1}{N} \sum_{n=1}^{N}\left|R_{\mathrm{lf}}[n]-R_{\text {exper }}[n]\right|^{2},
$$

where $N=5-10$ is the number of the first several spectral points of an experimental reflectivity spectrum.

The high-frequency asymptote $R_{\mathrm{hf}}(\omega)$ and the optical conductivity spectrum $\sigma_{\mathrm{KK}}(\omega)$, deduced by the anchor-window technique, are presented by dashed curves in Fig. 2. As seen, the anchor-window technique essentially improves an accuracy of the Kramers-Kronig analysis.

To check a reliability of the anchor-window technique, we measured the IR optical conductivity of fci-ZnMgRE quasicrystals by the spectroscopic ellipsometry technique. The measurements were carried out by a rotating compensator Fourier-transform ellipsometer IRSE (J.A. Woolam Co, Inc.). Results are presented by enlarged dots in Fig. 3. As seen, the KK-deduced optical conductivity values in the IR spectral range are close to the actual ones. This justifies a reliability of the suggested anchor-window technique.

\section{Acknowledgments}

The authors gratefully acknowledge a support by the Swedish Institute.

\section{References}

[1] A. Langsdorf, W. Assmus, J. Cryst. Growth 192, 152 (1998).

[2] K.-E. Peiponen, J.J. Saarinen, Rep. Prog. Phys. 72, 1 (2009).

[3] R.K. Ahrenkiel, J. Opt. Soc. Am. 61, 1651 (1971). 